\title{
Expression and clinical significance of cyclooxygenase 2 and survivin in human gliomas
}

\author{
FAN ZHANG ${ }^{*}$, JINJIN CHU* and FAN WANG \\ Department of Radiotherapy, The First Affiliated Hospital of Anhui Medical University, Hefei, Anhui 230022, P.R. China
}

Received September 17, 2015; Accepted January 26, 2017

DOI: $10.3892 / 01.2017 .6281$

\begin{abstract}
The present study aimed to determine cyclooxygenase 2 (COX-2) and survivin expression levels in glioma tissues, and to investigate their association with clinicopathological factors and patient survival. Immunohistochemistry was performed to evaluate COX-2 and survivin expression levels in paraffin-embedded surgically resected tissues from 70 patients with glioma and 7 individuals with normal brain tissues. The association between COX-2 and survivin expression levels and clinicopathological features was investigated using the $\chi^{2}$ test, and the survival time was analyzed using the Kaplan Meier method with log-rank test. COX-2 and survivin were overexpressed in glioma tissues, and higher expression levels were observed in glioma tissues of histological grades III-IV compared with those in grade I-II tumor tissues $(\mathrm{P}<0.05)$; however, the expression levels were not associated with gender, age, tumor size or location $(\mathrm{P}>0.05)$. There was a significant positive association between the expression levels of COX-2 and survivin in the glioma tissues. Additionally, COX-2 and survivin expression levels were significantly negatively correlated with the rate of survival. In conclusion, COX-2 and survivin expression is positively associated with the pathological grade of a glioma and may contribute to glioma tumorigenesis. Therefore, COX-2 and survivin may be sensitive predictors of a negative clinical prognosis for patients with glioma.
\end{abstract}

\section{Introduction}

Glioma derived from the neural epithelium is the most common type of primary brain tumor, accounting for $40-50 \%$ of all central nervous system neoplasms (1). Gliomas include

Correspondence to: Dr Fan Wang, Department of Radiotherapy, The First Affiliated Hospital of Anhui Medical University, 218 Jixi Road, Hefei, Anhui 230022, P.R. China

E-mail: wangfan1965@126.com

*Contributed equally

Key words: glioma, survivin, immunohistochemistry, prognosis, cyclooxygenase 2 astrocytomas, oligodendrogliomas, glioblastomas, ependymomas, medulloblastomas and glioblastoma multiforme (2). Glioma is genetically complex and is invasive to surrounding tissues, thus surgical resection is difficult (3). The recurrence of glioma following treatment is mostly due to the recurrence of the primary lesion (4). Although postoperative radiotherapy and chemotherapy may prolong survival times, the majority of patients with glioma live only 1-2 years after diagnosis (5).

Tumor occurrence, development, invasion and metastasis are complex processes involving multiple genes (6). Certain studies have indicated that non-steroidal anti-inflammatory drugs reduce the risk of various tumor types, for example, polyps of the large bowel in colorectal cancer tissues, and that cyclooxygenase (COX) may be associated with tumorigenesis (7). COX, also known as prostaglandin endoperoxidase $\mathrm{H}$ synthase, has two isoforms: COX-1, located in the cytoplasm and constitutively and stably expressed in various tissues, and COX-2, which is expressed at low levels or is not detectable in the majority of normal tissues, but can be induced in response to cell activation by growth factors, cytokines, inflammatory mediators and tumor promoters (8).

Survivin is a small but important member of the inhibitor-of-apoptosis protein family, with 142 amino acid residues and one baculovirus inhibitor of apoptosis protein repeat domain, which is a multifunctional protein that contributes to cellular homeostasis (9). Survivin is implicated in the inhibition of apoptosis and mitotic regulation, and promotes tumor development (10). Overexpression of survivin has been reported in cancer and is associated with a poor prognosis for a number of human malignancies, including non-small cell lung cancer (11). Thus, the present study determined the COX-2 and survivin expression levels in glioma tissues using immunohistochemistry methods, it examined the association between COX-2 and survivin, and it correlated the data with the clinicopathological characteristics of gliomas, including patient age, gender, tumor location, histopathological grade and patient survival.

\section{Materials and methods}

Specimen collection. Patients $(\mathrm{n}=70)$ with glioma were selected from The First Affiliated Hospital of Anhui Medical University (Hefei, Anhui, China) between October 2012 and December 2013; the patient's detailed clinicopathological characteristics were obtained from their medical files. The 
patients selected included 42 males and 28 females, with an age range of 7-70 years (median age 50 years). Patient characteristics are presented in Table I. The survival time was defined as the time between the day of the initial diagnosis and the day of patient mortality due to the glioma. Selected patients had no prior history of glioma. No patient received radiotherapy or chemotherapy prior to surgery and all patients were treated with surgery. The type of surgery patients' underwent was mainly according to the location and size of the tumor (for example total resection or subtotal resection). The tissue samples were obtained during the surgery. Surgically resected tissues were diagnosed by two pathologists according to World Health Organization (WHO) histological criteria (12). Tumors of grades I-II were of low malignancy and tumors graded III-IV were highly malignant. In addition, 7 normal brain tissues were derived from patients with arteriovenous malformation by arteriovenous malformation removal surgery. Written informed consent was obtained in all patients prior to enrollment in the present study. The study was approved by the Ethics Committee of The First Affiliated Hospital of Anhui Medical University.

Immunohistochemistry. Sections from formalin-fixed, paraffin-embedded tumor tissues (4- $\mu \mathrm{m}$ thick) were immunostained and assayed using a PV6000 polymer system (ZSGB-BIO, Beijing, China). All paraffin sections were heated to $90^{\circ} \mathrm{C}$ for $20 \mathrm{~min}$. Following routine deparaffinization by serial baths in xylene 3 times and dehydration in a graded series of ethanol (100, 95 and $80 \%)$ and distilled water, each section was treated with $0.3 \%$ hydrogen peroxide to block endogenous peroxidase activity. Sections were subsequently washed in $0.01 \mathrm{M}$ phosphate-buffered saline (PBS; pH 7.4), microwaved at $100^{\circ} \mathrm{C}$ for $20 \mathrm{~min}$ for antigen retrieval and then incubated overnight at $4^{\circ} \mathrm{C}$ with optimal dilutions of primary antibodies. Antibodies used were anti-COX-2 rabbit polyclonal antibody (dilution, 1:400; ab15191; Abcam, Cambridge, UK) and anti-survivin rabbit polyclonal antibody (dilution, 1:1,200; ab24479; Abcam). Following washing in PBS, the tissue sections were treated with secondary antibody, IgG-horseradish peroxidase polymer multimer (PV-6000; ZSGB-BIO, Beijing, China), at $37^{\circ} \mathrm{C}$ for $20 \mathrm{~min}$. Finally, the tissue sections were incubated with diaminobenzidine and counterstained with hematoxylin, cleared and mounted on neutralbalsam. In order to confirm COX-2 and survivin immunospecificity, 0.01 M PBS was supplied instead of primary antibody, as the negative control.

Evaluation. Stained tissue sections were observed under a light microscope. COX-2 and survivin expression levels were determined in 1,000 cells in 5 high-power fields for each tissue section. COX-2 staining in tumors was categorized as positive or negative and positive protein expression level was evaluated by two independent observers using a semi-quantitative immunoreactive score (IRS). Briefly, IRS was calculated by multiplication of staining intensity (graded as 0 , none; 1 , weak; 2 , moderate; and 3 , strong staining) and the percentage of positively-stained cells ( 0 , no stained cells; $1,1-10 \%$ stained cells; $2,11-50 \%$ stained cells; $3,51-80 \%$ stained cells and 4 , $81-100 \%$ stained cells). An IRS of $>3$ was considered to indicate a positive reaction (13).
Statistical analysis. SPSS version 19.0 was used to analyze data (IBM SPSS, Armonk, NY, USA). The $\chi^{2}$ test was performed to evaluate the COX-2 and survivin expression levels in patients with various clinicopathological characteristics. Spearman's correlation coefficient method was applied to evaluate the association between expressions levels of COX-2 and survivin. Survival analysis was estimated according to the Kaplan-Meier method from the date of diagnosis to the date of patient mortality due to the glioma. The difference in survival curves was examined by means of the log-rank test. $\mathrm{P}<0.05$ was considered to indicate a statistically significant difference.

\section{Results}

Immunohistochemistry results. In normal brain tissues, the COX-2 and survivin protein expression levels were negative. In tumor tissues, COX-2 expression was observed to be localized to the cytoplasm and nuclei of the tumor cells, whereas survivin expression was confined mostly to the nuclei of the tumor cells (Fig. 1). Positive COX-2 expression was identified in 44/70 glioma tissues, whereas positive survivin expression was observed in 39/70 glioma tissues.

Correlation with clinicopathological characteristics. COX-2 and survivin expression levels were correlated with pathological tumor grade. COX-2 expression occurred in $46.4 \%$ of low-grade malignancies and in $73.8 \%$ of high-grade tumors $(\mathrm{P}<0.05)$. Survivin was expressed in $39.3 \%$ of low-grade malignancies and $66.7 \%$ of high-grade tumors $(\mathrm{P}<0.05)$. COX-2 and survivin expression levels demonstrated a stepwise increase from weakly malignant (stage I-II) to highly malignant (stage III-IV) glioma; this was statistically significant, but expression was not correlated with gender, age, tumor size or location ( $\mathrm{P}>0.05$; Table I), indicating that these variables may not affect the expression levels of COX-2 and survivin.

Correlation between COX-2 and survivin. A total of 33 cases were identified as being COX-2- and survivin-positive, 20 were COX-2- and survivin-negative, 11 were $\mathrm{COX}$-2-positive and survivin-negative, and 6 were COX-2-negative and survivin-positive. There was a significant positive correlation between the expression level of COX-2 and survivin in the glioma tissues $(\mathrm{r}=0.50 ; \mathrm{P}<0.01)$.

Significant prognostic value of COX-2 and survivin. The follow-up of the patients ended in June 2015, and at this time, complete follow-up information was available for 60 patients. Kaplan-Meier survival analysis revealed that the median survival time for patients who were negative for COX-2 expression was 24 months, while those positive for COX-2 expression had a median survival time of 18 months. A log-rank test indicated that survival time for those positive for COX-2 was significantly lower compared with those negative for COX-2 $\left(\chi^{2}=10.113 ; \mathrm{P}<0.01\right.$; Fig. 2A). The median survival time the for patients who were negative for survivin expression was 24 months and that for patients positive for survivin expression was 16 months. A log-rank test confirmed that survivin expression was associated with a shorter survival time $\left(\chi^{2}=11.847\right.$; $\mathrm{P}<0.01$; Fig. 2B). Kaplan-Meier survival curves demonstrated 
Table I. COX-2 and survivin expression levels and clinicopathological factors.

\begin{tabular}{|c|c|c|c|c|c|c|c|}
\hline Clinicopathological factors & Cases, $\mathrm{n}$ & COX-2, n (\%) & $\chi^{2}$ & P-value & Survivin, n (\%) & $\chi^{2}$ & P-value \\
\hline \multicolumn{8}{|l|}{ Gender } \\
\hline Male & 42 & $28(66.7)$ & \multirow[t]{2}{*}{0.653} & \multirow[t]{2}{*}{$>0.05$} & $25(59.5)$ & \multirow[t]{2}{*}{0.618} & \multirow[t]{2}{*}{$>0.05$} \\
\hline Female & 28 & $16(57.1)$ & & & $14(50.0)$ & & \\
\hline \multicolumn{8}{|l|}{ Age, years } \\
\hline$\geq 50$ & 46 & $26(56.5)$ & \multirow[t]{2}{*}{2.307} & \multirow[t]{2}{*}{$>0.05$} & $23(50.0)$ & \multirow[t]{2}{*}{1.776} & \multirow[t]{2}{*}{$>0.05$} \\
\hline$<50$ & 24 & $18(52.9)$ & & & $16(66.7)$ & & \\
\hline \multicolumn{8}{|l|}{ Pathological WHO grade } \\
\hline I-II & 28 & $13(46.4)$ & \multirow[t]{2}{*}{5.395} & \multirow[t]{2}{*}{$<0.05$} & $11(39.3)$ & \multirow[t]{2}{*}{5.105} & \multirow[t]{2}{*}{$<0.05$} \\
\hline III-IV & 42 & $31(73.8)$ & & & $28(66.7)$ & & \\
\hline \multicolumn{8}{|l|}{ Tumor size, $\mathrm{cm}$} \\
\hline$\geq 5$ & 43 & $28(65.1)$ & \multirow[t]{2}{*}{0.244} & \multirow[t]{2}{*}{$>0.05$} & $24(55.8)$ & \multirow[t]{2}{*}{0.000} & \multirow[t]{2}{*}{$>0.05$} \\
\hline$<5$ & 27 & $16(59.3)$ & & & $15(55.6)$ & & \\
\hline \multicolumn{8}{|l|}{ Tumor position } \\
\hline Frontal lobe & 28 & $19(67.9)$ & \multirow[t]{5}{*}{3.586} & \multirow[t]{5}{*}{$>0.05$} & $17(60.7)$ & \multirow[t]{5}{*}{2.530} & \multirow[t]{5}{*}{$>0.05$} \\
\hline Temporal lobe & 19 & $14(73.7)$ & & & $12(63.2)$ & & \\
\hline Parietal lobe & 10 & $5(50.0)$ & & & $5(50.0)$ & & \\
\hline Occipital lobe & 7 & $3(42.9)$ & & & $3(42.9)$ & & \\
\hline Epencephala & 6 & $3(50.0)$ & & & $2(33.3)$ & & \\
\hline
\end{tabular}

COX-2, cyclooxygenase 2; WHO, World Health Organization.

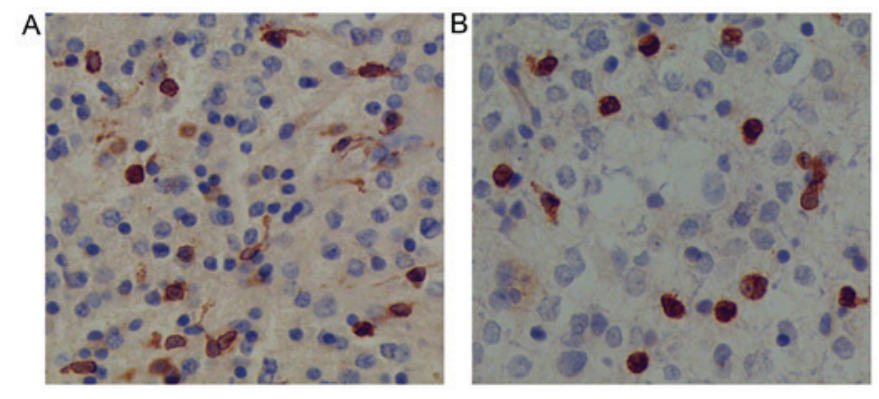

Figure 1. Immunohistochemical staining for (A) cyclooxygenase 2 and (B) survivin in glioma tissues (x400 magnification).

that COX-2 (III-IV; $\chi^{2}=4.252 ; \mathrm{P}<0.05$; Fig. 3A) and Survivin (III-IV; $\chi^{2}=4.571 ; \mathrm{P}<0.05$; Fig. 3B) expression levels were significant prognostic predictors for high-grade malignant glioma. Additionally, COX-2 (I-II; $\chi^{2}=5.069$; P<0.05; Fig. 4A) and Survivin (I-II; $\chi^{2}=4.709 ; \mathrm{P}<0.05$; Fig. 4B) expression levels were significant prognostic predictors for low-grade malignant glioma. COX-2 and survivin expression levels were significantly associated with poor survival. The 2-year survival rate for patients who were negative for COX-2 and survivin expression was significantly higher compared with that for patients who were positive for COX-2 and survivin expression (58.3 vs. $22.5 \% ; \chi^{2}=12.156 ; \mathrm{P}<0.05$; Fig. 5). Patients with grade IV glioma who were negative for COX-2 and survivin expression experienced a median survival time of 24 months, and this was significantly longer compared with the survival time for patients who were positive for COX-2 and survivin expression (14 months; $\chi^{2}=3.933 ; \mathrm{P}<0.05$ ).

\section{Discussion}

Abnormal expression levels of apoptotic genes perturbs apoptosis, allowing cells to escape normal control mechanisms, and inducing cell cycle disorders and tumorigenesis (14). COX-2 and survivin have been suggested to be involved in tumorigenesis $(15,16)$, thus they are of interest as potential therapeutic targets. Currently, pathological diagnosis, in particular WHO tumor grade guidelines, are important for predicting prognosis in those patients with gliomas. Therefore, the present study determined COX-2 and survivin expression levels, and evaluated their utility as prognostic markers for glioma.

COX-2 is an inducible enzyme involved in the conversion of arachidonic acid to prostaglandin $\mathrm{H} 2$, which is subsequently converted by specific prostaglandin synthases to prostaglandin E2 (17). Increasing levels of prostaglandin E2 (main product of COX-2) promotes cell proliferation, invasion and angiogenesis, and inhibits apoptosis, a feature closely associated with tumorigenesis (18). Previous studies have suggested that positive staining for COX-2 is associated with cancer, thus it is used as a diagnostic and therapeutic target for breast, colon, gastric and esophageal cancer (19-21). Elevated COX-2 expression levels in tumors are associated with the inhibition of apoptosis, increased cell proliferation and differentiation, increased angiogenesis, tumor invasion and metastasis, and the inhibition of cell immune function (22). Temel and Kahveci (23) reported that glial cells did not express COX-2 in normal brain tissues and that COX-2 protein expression was observed predominantly in all glioma types; its immunoreactivity was upregulated in high-grade malignancies, particularly in glioblastoma multiforme (grade IV). COX-2 has also been 

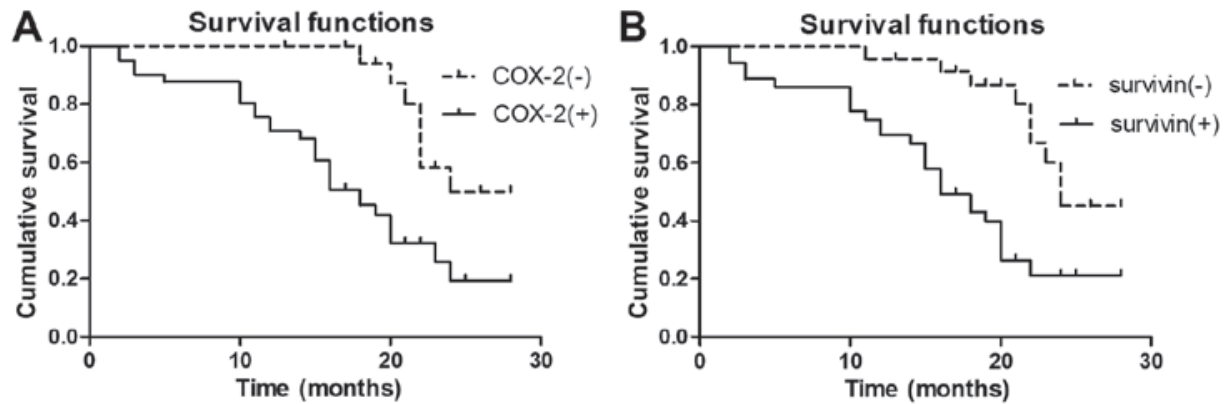

Figure 2. Kaplan-Meier survival plots defined by (A) COX-2 and (B) survivin expression in patients with glioma tumor grades I-IV. COX-2, cyclooxygenase 2 .
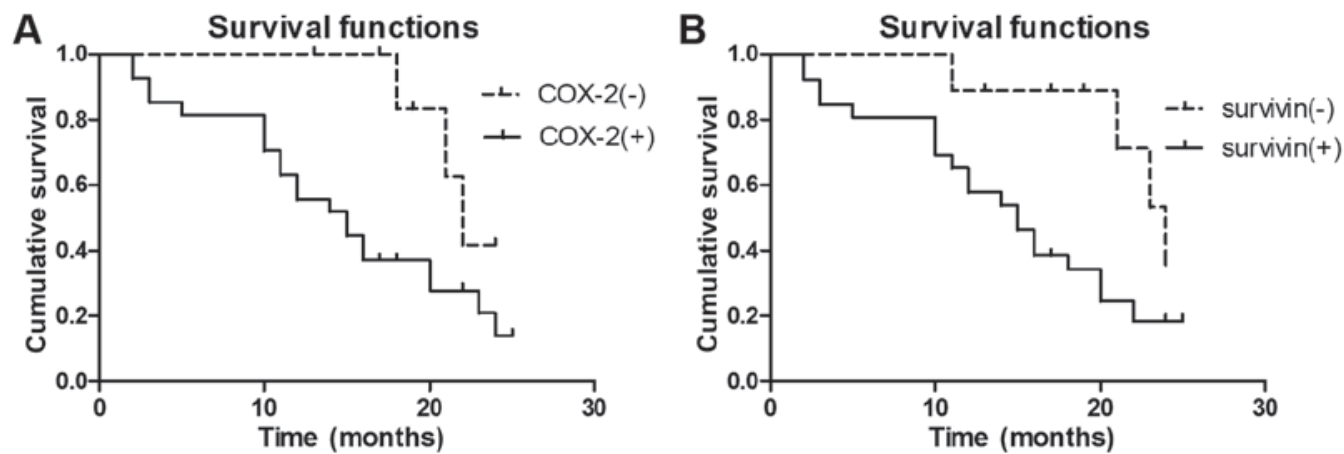

Figure 3. Kaplan-Meier survival plots defined by (A) COX-2 and (B) survivin expression in patients with glioma tumor grades III-IV. COX-2, cyclooxygenase 2 .
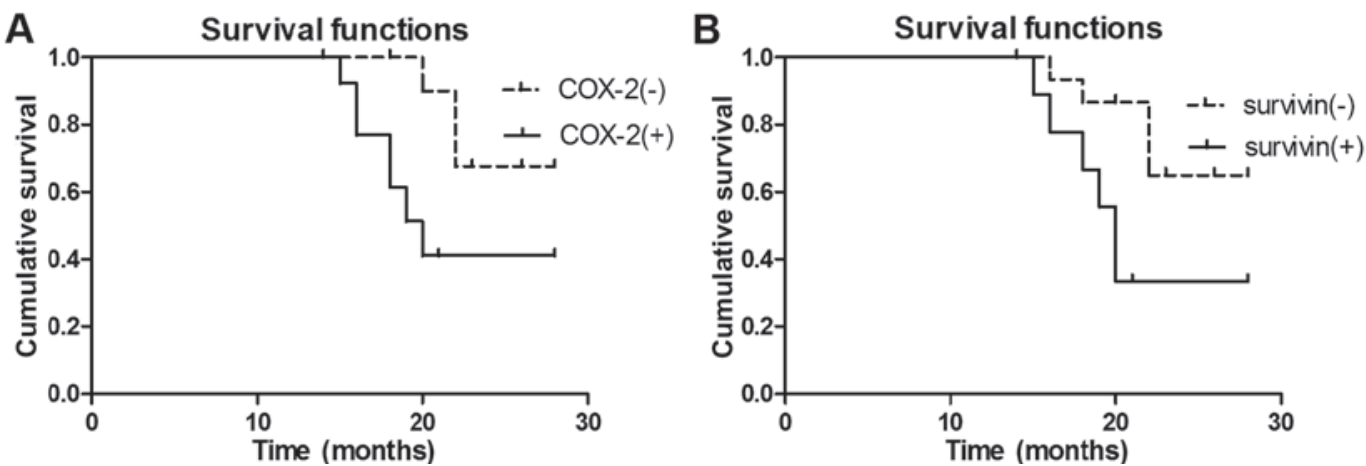

Figure 4. Kaplan-Meier survival plots defined by (A) COX-2 and (B) survivin expression in patients with glioma tumor grades I-II. COX-2, cyclooxygenase 2 .

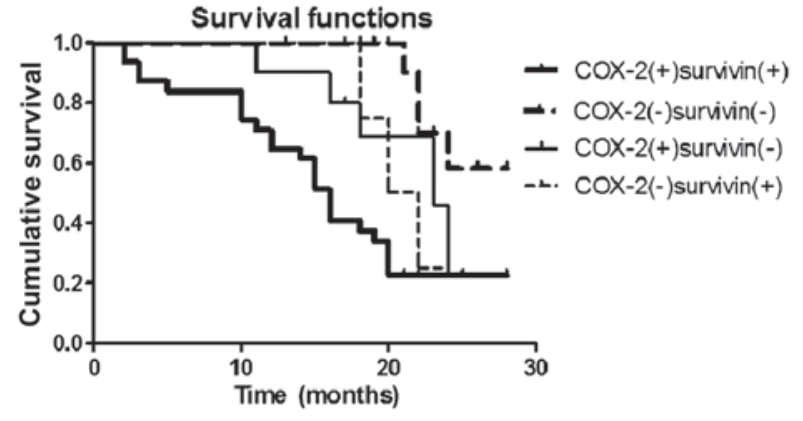

Figure 5. Kaplan-Meier survival plot of four groups defined by positive/negative COX-2 and positive/negative survivin expression. COX-2, cyclooxygenase 2 .

confirmed to be present in the surrounding tissues of necrotic tumor areas in glioblastoma multiforme (23). The present study demonstrated that COX-2 expression was detected in 13/28 (46.4\%) low-grade malignant gliomas and in 31/42 (73.8\%) high-grade malignant gliomas. COX-2 overexpression in gliomas and a stepwise increase in COX-2 immunoreactivity from weakly to highly malignant gliomas occurred, and this was statistically significant. El-Sayed and Taha (24) revealed that COX-2 expression was significantly associated with poor survival $(r=0.58 ; \mathrm{P}<0.001)$. The follow-up data of the present study demonstrated that patients with COX-2-positive tumors had a significantly shorter survival time compared with patients who were negative for COX-2 (18 vs. 24 months). Furthermore, COX-2 may be used to predict prognosis in highand low-grade glioma types. Consequently, COX-2 expression may be a significant negative predictor of prognosis for glioma.

Survivin is an inhibitor of apoptosis that has other properties that modulate mitosis, apoptosis and the cellular stress response (25). Survivin is expressed during embryonic and 
fetal development, and is found in cancer cells (including hepatocellular carcinoma and gastrointestinal cancer), but not in adult terminal differentiated tissues (26). Survivin enhances tumor cell survival primarily by suppressing apoptosis via the direct inhibition of caspase-associated proteins (27). Survivin is expressed during the $\mathrm{G}_{2} / \mathrm{M}$ phase of the cell cycle and is a member of the chromosomal passenger complex, a regulator of chromosome-microtubule attachment, spindle assembly checkpoint and cytokinesis at cell division (28). Therefore, survivin functions as a key regulator of chromosomal segregation and cytokinesis, repressing cell death (29). A previous study of patients with glioblastoma indicated that survivin was associated with patient prognosis (30). While cytoplasmic survivin did not modify prognosis, nuclear survivin localization was correlated with a significantly lower survival rate compared with patients with low nuclear survivin levels. The present study observed that survivin accumulated in the nuclei of glioma cells, thus it may be useful for the survival prognosis. Chakravarti et al (31) demonstrated a negative association between survivin expression and the survival of glioma patients. Kogiku et al (32) also observed a significant association between survivin and age, Karnofsky performance scale (KPS) score and grade $(\mathrm{P}=0.0017, \mathrm{P}=0.0006$ and $\mathrm{P}=0.0002$, respectively). This indicated that older patients with glioblastoma multiforme (grade IV) and a lower KPS score tended to have a higher survivin expression level. In addition, the median survival time of patients with high survivin expression was significantly shorter compared with that for patients with low expression (322 vs. 1,084 days) (32). In the present study, survivin expression was detected in 11/28 (39.3\%) low-grade glioma tissues and in 28/42 (66.7\%) high-grade glioma tissues. Survivin immunoreactivity was upregulated significantly in high-grade glioma, but it was not correlated with gender, age, tumor size or location. In the follow-up data, patients with survivin-positive tumors experienced a significantly shorter survival time compared with those who were negative for survivin (16 vs. 24 months), and this was in agreement with previous studies $(31,32)$. Furthermore, survivin may be used to predict prognosis for low-grade and high-grade gliomas.

Therefore, COX-2 and survivin proteins may be important for changes in malignancy during glioma tumorigenesis. A study by Mehar et al (33) indicated that survivin expression could be induced by celecoxib and that it was dependent on COX-2 expression. A significant immunoreactive association between COX-2 and survivin protein was observed in ovarian cancer (34) and endometrial carcinoma (35). Thus, COX-2 may act as an upstream regulatory factor, with high expression of COX-2 upregulating survivin by preventing survivin ubiquitination and proteasome degradation. To the best of our knowledge, this interaction has not yet been investigated in glioma.

The present study demonstrated a significant positive correlation between the expression of COX-2 and survivin in glioma, and hypothesized that COX-2 and survivin may facilitate tumor occurrence and progression via a common signaling pathway. The two proteins were potent indicators of glioma survival, and follow-up data revealed that the survival time for patients who were positive for the two proteins was significantly lower compared with patients who were negative for the two proteins. This significant correlation of
COX-2 and survivin may further highlight the advantage of a combinational evaluation with COX-2 and survivin to determine the prognosis in patients with glioma. Furthermore, the significantly longer survival time of patients diagnosed with grade IV glioma without the expression of COX-2 and survivin suggested an advantage of combining a pathological diagnosis with the expression of COX-2 and survivin. Ren et al (36) confirmed that inhibition of proliferation and promotion of apoptosis in U251 glioma cells could be induced by celecoxib in a dose- and time-dependent manner, and that this may occur by downregulation of survivin expression. However, the specific signal transduction pathway by which this may occur in glioma remains unknown. Thus, the link between COX-2 and survivin requires further investigation. Furthermore, these proteins may have potential value for glioma therapy, as COX-2 inhibitors are effective for reducing the risk of malignancies and inhibiting tumorigenesis (37). Rödel et al (38) revealed that a drug to block survivin activity by downregulation may inhibit tumor proliferation.

There are certain limitations to the present study. Firstly, the patients underwent surgery using a variety of methods and surgeons. Also, the tissue specimens were evaluated by different pathologists, although all tissues were re-examined based on strict criteria (WHO guidelines). Furthermore, the sample size was limited, which may have obscured significant findings. A study with a larger sample size is required. A multi-institutional study of patient populations in Anhui or all of China would aid in improving our current understanding of the prognosis of glioma patients using COX-2 and survivin expression analysis.

Despite the aforementioned factors, the results of the present study may aid in defining the tumorigenic mechanism underlying glioma and assist with determining an effective diagnosis and prognosis. The present study provided convincing evidence for the specific accumulation of COX-2 and survivin existing in glioma tissues, suggesting that they serve an important role in the process of carcinogenesis. The present study suggested that COX-2 and survivin are associated with the severity of glioma, as patients who were positive for the expression of the two proteins demonstrated shorter survival times. This may aid the establishment of a comprehensive therapeutic evaluation of glioma, and these proteins may have potential and suitable uses for targeted therapy. Further studies are required in order to investigate these findings. Additionally, COX-2 and survivin proteins may be useful for biomarker studies to predict glioma severity and patient prognosis.

\section{Acknowledgements}

The present study was supported by the Anhui Provincial Natural Science Foundation (grant no. 1308085MH134).

\section{References}

1. Holland EC: Glioblastoma multiforme: The terminator. Proc Natl Acad Sci USA 97: 6242-6244, 2000.

2. Louis DN, Pomeroy SL and Cairncross JG: Focus on central nervous system neoplasia. Cancer Cell 1: 125-128, 2002.

3. Nakada M, Nakada S, Demuth T, Tran NL, Hoelzinger DB and Berens ME: Molecular targets of glioma invasion. Cell Mol Life Sci 64: 458-78, 2007. 
4. Omay SB and Vogelbaum MA: Current concepts and newer developments in the treatment of malignant gliomas. Indian J Cancer 46: 88-95, 2009.

5. Lima FR, Kahn SA, Soletti RC, Biasoli D, Alves T, da Fonseca AC, Garcia C, Romão L, Brito J, Holanda-Afonso R, et al Glioblastoma: Therapeutic challenges, what lies ahead. Biochim Biophys Acta 1826: 338-349, 2012.

6. Altieri DC: Survivin, cancer networks and pathway-directed drug discovery. Nat Rev Cancer 8: 61-70, 2008.

7. Ke HL, Tu HP, Lin HH, Chai CY, Chang LL, Li WM, Li CC Lee YC, Yeh HC, Wu WJ and Bau DT: Cyclooxygenase-2 (COX-2) up-regulation is a prognostic marker for poor clinical outcome of upper tract urothelial cancer. Anticancer Res 32: 4111-4116, 2012

8. Cebola I and Peinado MA: Epigenetic deregulation of the COX pathway in cancer. Prog Lipid Res 51: 301-313, 2012.

9. Rödel F, Reichert S, Sprenger T, Gaipl US, Mirsch J, Liersch T, Fulda S and Rödel C: The Role of Survivin for Radiation Oncology: Moving Beyond Apoptosis Inhibition. Curr Med Chem 18: 191-199, 2011.

10. Kanwar JR, Kamalapuram SK and Kanwar RK: Targeting survivin in cancer: Patent review. Expert Opin Ther Pat 20: 1723-1737, 2010.

11. Chen XQ, Yang S, Li ZY, Lu HS, Kang MQ and Lin TY: Effects and mechanism of downregulation of survivin expression by RNA interference on proliferationand apoptosis of lung cancer cells. Mol Med Rep 5: 917-922, 2012.

12. Louis DN, Ohgaki H, Wiestler OD, Cavenee WK, Burger PC, Jouvet A, Scheithauer BW and Kleihues P: The 2007 WHO classification of tumours of the central nervous system. Acta Neuropathol 114: 97-1099, 2007.

13. Remmele W and Stegner HE: Recommendation for uniform definition of an immunoreactive score (IRS) for immunohistochemical estrogen receptor detection (ER-ICA) in breast cancer tissue. Pathologe 8: 138-140, 1987 (In German).

14. Fesik SW: Promoting apoptosis as a strategy for cancer drug discovery. Nat Rev Cancer 5: 876-885, 2005.

15. Trifan OC and Hla T: Cyclooxygenase- 2 modulates cellular growth and promotes tumorigenesis. J Cell Mol Med 7: 207-222, 2003.

16. Altieri DC: Survivin, versatile modulation of cell division and apoptosis in cancer. Oncogene 22: 8581-8589, 2003.

17. Grösch S, Maier TJ, Schiffmann S and Geisslinger $G$ : Cyclooxygenase-2 (COX-2)-independent anticarcinogenic effects of selective COX-2 inhibitors. J Natl Cancer Inst 98: 736-47, 2006.

18. Kim YM, Shin YK, Jun HJ, Rha SY and Pyo H: Systematic analyses of genes associated with radiosensitizing effect by celecoxib, a specific cyclooxygenase-2 inhibitor. Radiat Res 52 : 752-765, 2011.

19. Hoellen F, Kelling K, Dittmer C, Diedrich K, Friedrich M and Thill M: Impact of cyclooxygenase-2 in breast cancer. Anticancer Res 31: 4359-4367, 2011.

20. Moreira L and Castells A: Cyclooxygenase as a target for colorectal cancer chemoprevention. Curr Drug Targets 12: 1888-1894, 2011.

21. Zimmermann KC, Sarbia M, Weber AA, Borchard F, Gabbert HE and Schrör K: Cyclooxygenase-2 expression in human esophageal carcinoma. Cancer Res 59: 198-204, 1999.
22. Greenhough A, Smartt HJ, Moore AE, Roberts HR, Williams AC, Paraskeva C and Kaidi A: The COX-2/PGE 2 pathway: Key roles in the hallmarks of cancer and adaptation to the tumour microenvironment. Carcinogenesis 30: 377-386, 2009.

23. Temel SG and Kahveci Z: Cyclooxygenase-2 expression in astrocytes and microgliain human oligodendroglioma and astrocytoma. J Mol Histol 40: 369-377, 2009.

24. El-Sayed M and Taha MM: Immunohistochemical expression of cycloxygenase-2 in astrocytoma: Correlation with Angiogenesis, tumor progression and survival. Turk Neurosurg 21: 27-35, 2011.

25. Altieri DC: Targeting survivin in cancer. Cancer Lett 332 225-228, 2013.

26. Liu W, Zhu F, Jiang Y, Sun D, Yang B and Yan H: SiRNA targeting survivin inhibits the growth and enhances the chemosensitivity of hepatocellularcarcinoma cells. Oncol Rep 29: 1183-1188, 2013

27. Mobahat M, Narendran A and Riabowol K: Survivin as a preferential target for cancer therapy. Int J Mol Sci 15: 2494-2516, 2014.

28. Jeyaprakash AA, Klein UR, Lindner D, Ebert J, Nigg EA and Conti E: Structure of a Survivin-Borealin-INCENP core complex reveals how chromosomal passengers travel together. Cell 131: 271-285, 2007.

29. Altieri DC: Survivin-The inconvenient IAP. Semin Cell Dev Biol 39: 91-96, 2015.

30. Shirai K, Suzuki Y, Oka K, Noda SE, Katoh H, Suzuki Y, Itoh J, Itoh $\mathrm{H}$, Ishiuchi $\mathrm{S}$, Sakurai $\mathrm{H}$, et al: Nuclear survivin expression predicts poorer prognosis in glioblastoma. J Neurooncol 91: 353-358, 2009.

31. Chakravarti A, Noll E, Black PM, Finkelstein DF, Finkelstein DM, Dyson NJ and Loeffler JS: Quantitatively determined survivin expression levels are of prognostic value in human gliomas. Oncol 20: 1063-1068, 2002.

32. Kogiku M, Ohsawa I, Matsumoto K, Sugisaki Y, Takahashi H, Teramoto A and Ohta S: Prognosis of glioma patients by combined immunostaining for survivin, Ki-67 and epidermal growth factor receptor. J Clin Neurosci 15: 1198-1203, 2008.

33. Mehar A, Macanas-Pirard P, Mizokami A, Takahashi Y, Kass GE and Coley HM: The effects of cyclooxygenase-2 expression in prostate cancer cells: Modulation of response to cytotoxic agents. J Pharmocol Exp Ther 324: 1181-1187, 2008.

34. Athanassiadou P, Grapsa D, Athanassiades P, Gonidi M, Athanassiadou AM, Tsipis A and Patsouris E: The prognostic significance of COX-2 and survivin expression in ovarian cancer. Pathol Res Pract 204: 241-249, 2008.

35. Erkanli S, Bolat F, Kayaselcuk F, Demirhan B and Kuscu E: COX-2 and survivin are overexpressed and positively correlated in endometrial carcinoma. Gynecol Oncol 104: 320-325, 2007.

36. Ren Z, Zhang LZ and Wang RZ: Effect of celecoxib on proliferation, apoptosis and Survivin expression in human glioma cell line U251. Chin J Can 29: 294-299, 2010.

37. Schetter AJ, Heegaard NH and Harris CC: Inflammation and cancer: Interweaving micro RNA, free radical, cytokine and p53 pathways. Carcinogenesis 31: 37-49, 2010.

38. Rödel F, Frey B, Leitmann W, Capalbo G, Weiss C and Rödel C: Survivin antisense oligonucleotides effectively radiosensitize colorectal cancer cells in both tissues culture and murine xenograft models. Int J Radiat oncol Biol Phys 71: 247-255, 2008. 